\title{
Abordagens pedagógicas, Estratégias de ensino-aprendizagem e Modalidades de uso de Tecnologias de Informação e Comunicação: uma Análise de Cursos para Formação Continuada de Professores de Ciências
}

Rosilaine de Fátima Wardenski rosiwardenski@gmail.com 0000-0002-3026-2971 Universidade Federal do Rio de Janeiro, Rio de Janeiro, Rio de Janeiro, Brasil.

Miriam Struchiner miriamstru@gmail.com 000-0002-9979-2364 Universidade Federal do Rio de Janeiro, Rio de Janeiro, Rio de Janeiro, Brasil.

Tais Rabetti Giannella taisrg@yahoo.com.br Universidade Federal do Rio de Janeiro Rio de Janeiro, Rio de Janeiro, Brasil.

\section{RESUMO}

Este trabalho objetiva analisar ambientes virtuais de aprendizagem voltados para a formação continuada de professores de ciências. Utilizando-se um modelo de análise de materiais educativos baseados na Internet para o ensino de ciências, foram analisadas as abordagens pedagógicas, as estratégias de ensino/aprendizagem e as modalidades de uso das TICs de seis cursos. Cinco cursos apresentaram abordagens pedagógicas centradas nos alunos/aprendizagem, sendo identificadas as estratégias: aprender explorando (C2, C5 e C 6), aprender fazendo ( $C 1, C 2, C 5$ e $C 6)$, aprender refletindo $(C 1, C 4, C 5$ e $C 6)$ e aprendizagem incidental (C6) . Um curso apresentou abordagem centrada no professor/ensino. Em relação ao uso das TICs, identificamos as modalidades: Acesso e organização da informação; Atividades autênticas e Aprendizagem colaborativa. Conclui-se que a integração de TICs na formação de professores pode potencializar o uso de abordagens para aprendizagem ativa e colaborativa, contribuindo para a melhoria do ensino de ciências.

PALAVRAS-CHAVE: Análise de ambientes virtuais de aprendizagem. Formação continuada de professores de ciências. Tecnologias de informação e comunicação. 


\section{INTRODUÇÃO}

$\mathrm{Na}$ literatura, diversos artigos relatam experiências de formação continuada de professores de ciências, com o uso de Tecnologias de Informação e Comunicação (TICS), e reforçam a importância de se fortalecer parcerias entre universidades e escolas (CICILLINI e SILVA, 2005; LIMA E PINHEIRO, 2005; ZAPAROLI, BUENO e ARRUDA, 2005). De uma maneira geral, estes trabalhos apontam que as TICs podem constituir-se em importantes aliadas na promoção de iniciativas de extensão universitária semipresenciais e a distância, tendo em vista a oferta de uma riqueza de bases de informações, diferentes formas de representação do conhecimento que facilitam a compreensão de conceitos e fenômenos difíceis de serem visualizados, a experimentação sem os riscos da vida real como é o caso das simulações e a comunicação entre os diferentes atores do processo educativo (NAVAS, MALACHIAS, NUNES e FEJES, 2005; NOVELLO, LAURINO e RODRIGUES, 2005).

Se por um lado há um reconhecimento do potencial de democratização do conhecimento promovido pelo uso das TICs, diversos autores chamam a atenção para o caráter de aligeiramento e precarização da formação viabilizada por muitas destas iniciativas (BARRETO, 2004). Iniciativas que em geral desconsideram as vozes e experiências dos professores e oferecem modelos verticalizados e pouco dialógicos, baseados na visão de que os centros produtores de conhecimento (como as universidades) devem transmitir informações às escolas e seus professores (CICILLINI e SILVA, 2009; GABINI e DINIZ, 2007).

Partindo do pressuposto de que não são as tecnologias em si que promovem modelos educativos inovadores, mas as abordagens pedagógicas adotadas e a visão de formação implicadas nesse processo, este trabalho discute como as TICs vem sendo utilizadas na oferta de cursos de formação de professores da educação básica.

Em especial, tem como objetivo analisar os ambientes virtuais de aprendizagem de seis cursos oferecidos por docentes universitários, voltados para a formação continuada de professores de ciências, enfocando-se suas abordagens pedagógicas, estratégias de ensino-aprendizagem e modalidades de uso das TICs.

Este trabalho se situa no contexto de um projeto de Extensão Universitária (STRUCHINER e GIANNELLA, 2010) que oferece apoio pedagógico e tecnológico a professores da área das ciências e da saúde na implementação de cursos semipresenciais ou a distância. A partir da disponibilização de uma ferramenta de autoria de cursos na Internet (Constructore) e da possibilidade de parceria no planejamento, acompanhamento e avaliação dos cursos, o projeto visa contribuir com o estreitamento das relações universidade-sociedade, aproveitando o potencial das TICs para a difusão e construção coletiva de conhecimentos.

A presente análise pauta-se na compreensão de que os Ambientes Virtuais de Aprendizagem (AVAs) podem constituir-se em lentes por meio das quais podemos pesquisar e desenvolver conhecimentos sobre o processo educativo (GIANNELLA \& STRUCHINER, 2010), tendo em vista que estas ferramentas podem ser adaptadas de acordo com o contexto de ensino, os objetivos, as necessidades e as metodologias dos docentes envolvidos (MISHRA \& KOHELER, 2008; ESPINDOLA, 2010). 


\section{REVISÃO DA LITERATURA}

A literatura apresenta diversos trabalhos que caracterizam iniciativas de formação continuada semipresenciais (CICILLINI e SILVA, 2009; GEREMIAS, CIMA e ANGOTTI, 2005; NOVELLO, LAURINO e RODRIGUES, 2005; TESSIO, REMBADO, SANTOS e PORRO, 2005) ou a distancia (LIMA e PINHEIRO, 2005; MENDEZ \& FIGUEIROA, 2005) voltadas para professores de ciências da educação básica.

De uma maneira geral, esses trabalhos partem da visão de que o professor é o principal agente de mudanças nos processos de ensino-aprendizagem e reforçam o papel das tecnologias para a ampliação das oportunidades de formação (CICILLINI e SILVA, 2009; GABINI e DINIZ, 2007; TESSIO, REMBADO, SANTOS e PORRO, 2005). Os autores defendem um aprendizado pautado na problematização da realidade e na construção do conhecimento pelos alunos, o que leva à necessidade de atualização do professor, que deve aprimorar constantemente suas práticas de ensino. Nesse sentido, Aires \& Lambach (2009) afirmam que os professores devem se apropriar das discussões referentes a mudanças nas estratégias didáticas, sendo que este processo não pode estar desvinculado de seu cotidiano, de maneira que possam compreender e aplicar novas metodologias com base no contexto escolar e nos objetivos relacionados à aprendizagem dos estudantes.

Observa-se, assim, que diversos trabalhos que descrevem iniciativas de formação continuada de professores de ciências defendem, em geral, abordagens pedagógicas centradas mais no aluno/na aprendizagem do que no professor/conteúdo (KEMBER e KWAN, 2000), de maneira a promover espaços de reflexão-ação pautados na problematização e avaliação de seus cotidianos de ensino e na formulação de possíveis estratégias de mudança (CICILLINI e SILVA, 2009; GABINI e DINIZ, 2007; TESSIO, REMBADO, SANTOS e PORRO, 2005). Ao descreverem uma iniciativa de formação continuada de professores, Tessio et al (2005), por exemplo, destacam a colaboração entre diversos sujeitos como estratégia para promover melhorias no processo educativo. Segundo os autores, muitos professores (alunos do curso descrito), estavam inseridos na prática de sala de aula há muitos anos e apresentavam dificuldades na leitura e interpretação de textos, o que dificultava a compreensão de materiais que trazem propostas de estratégias didáticas inovadoras. Diante desse quadro, foi utilizado o trabalho colaborativo com o objetivo de facilitar a compreensão dos textos, auxiliando os professores na leitura de publicações com conteúdo pedagógico, e na reflexão acerca da utilização do método científico em sala de aula (TESSIO, REMBADO, SANTOS e PORRO, 2005).

A partir da concepção de que o aluno deve ser o centro do processo educativo, os artigos citados descreviam o planejamento e o desenvolvimento colaborativo de projetos, materiais e atividades didáticas (AIRES e LAMBACH, 2009; ARROIO DINIZ e GIORDAN, 2005; CICILLINI e SILVA, 2009; GABINI e DINIZ, 2005; 2007; GEREMIAS, CIMA e ANGOTTI, 2005; MENDEZ e FIGUEIROA, 2005; NOVELLO, LAURINO e RODRIGUES, 2005; REIS, SOUZA e BISCH, 2007). Dentre esses, os cursos relatados nos trabalhos de Aires e Lambach (2009) e Cicillini e Silva (2009), incluíam ainda a realização de reuniões e reflexões conjuntas, com o objetivo de identificar as principais necessidades formativas dos participantes dos cursos. A partir dessas 
a fim de visualizar as possibilidades de conexões entre diversas áreas do conhecimento para tratar temas previamente delimitados.

A fim de servir como base para a realização dessas atividades, os cursos utilizavam diversas estratégias de ensino-aprendizagem, como: a) disponibilização de recursos de informação em diferentes formatos, como textos, áudio-visuais, gráficos e indicações de bibliografias e outras fontes, com o objetivo de servir como fundamentação teórica(AIRES e LAMBACH, 2009; CICILLINI e SILVA, 2009; GABINI e DINIZ, 2007; GEREMIAS, CIMA e ANGOTTI, 2005; MENDEZ \& FIGUEIROA, 2005; REIS, SOUZA e BISCH, 2007); b) realização de discussões em grupo, permitindo a troca de experiências e informações, e possibilidades de atividades a serem realizadas (AIRES e LAMBACH, 2009; ARROIO DINIZ e GIORDAN, 2005; GABINI \& DINIZ, 2005; 2007; LIMA e PINHEIRO, 2005; MENDEZ e FIGUEIROA, 2005; NOVELLO, LAURINO e RODRIGUES, 2005; REIS, SOUZA e BISCH, 2007); e c) elaboração de registros e reflexões sobre o processo de aprendizagem, para dar aos alunos a oportunidade de organizar o pensamento, além de manter uma documentação acerca dos conhecimentos construídos ao longo do curso, que pudessem ser acessados posteriormente (MENDEZ e FIGUEIROA, 2005; REIS, SOUZA e BISCH, 2007).

No que diz respeito às modalidades pedagógicas de uso das TICs em iniciativas de formação docente, diversos trabalhos defendem uma visão crítica de apropriação das tecnologias, de maneira que a vivência experienciada nos cursos possa repercutir em práticas educativas inovadoras (GABINI e DINIZ, 2005; 2007; TESSIO, REMBADO, SANTOS e PORRO, 2007).

Observa-se que os recursos tecnológicos utilizados com mais frequência nos cursos são aqueles relacionados ao compartilhamento e organização de materiais informativos que, segundo Mayes e Fowler (1999), contribuem para o estágio de aprendizagem de conceituação ou fixação de conteúdos de ensino. Um exemplo é o trabalho relatado por Mendez \& Figueiroa (2005), no qual leituras disponibilizadas online serviam como informações adicionais e suporte teórico para auxiliar os participantes do curso no planejamento e reflexão acerca de suas atividades. Outros trabalhos relatam a utilização de ferramentas que permitiam aos alunos compartilhar materiais, registros de atividades e práticas pedagógicas desenvolvidas (ARROIO, DINIZ e GIORDAN, 2005; CICILLINI e SILVA, 2009).

Buscando valorizar a troca de experiências e a construção coletiva do conhecimento, muitos cursos lançam mão do potencial das ferramentas de comunicação, tais como fóruns de discussão e chat (LIMA e PINHEIRO, 2005; MENDEZ e FIGUEIROA, 2005; NOVELLO, LAURINO e RODRIGUES, 2005). Tendo como base o pressuposto de que a oportunidade de registrar e compartilhar experiências com os colegas facilita a melhoria das ações pedagógicas do professor, Lima \& Pinheiro (2005) relatam a utilização dos recursos comunicacionais com o objetivo de criar comunidades pedagógicas on line. Já no trabalho relatado por Mendez \& Figueiroa (2005), o fórum de discussão foi utilizado para os professores/alunos apresentarem idéias e sugestões para o enfrentamento de uma situação-problema e compartilharem seus planejamentos didáticos. Segundo os autores, o objetivo desta atividade era estimulá-los a buscar soluções para determinados problemas e a construir juntos novas metodologias de ensino. 
A partir da perspectiva de que a formação necessita estar articulada com o cotidiano de trabalho do professor, diversos autores defendem que os indivíduos devem se apropriar do processo de desenvolvimento de estratégias e materiais de ensino inovadores. (CICILLINI e SILVA, 2009; GABINI e DINIZ, 2005; REIS, SOUZA e BISCH, 2007; TESSIO, REMBADO, SANTOS e PORRO, 2005). Os trabalhos apresentados por esses autores relatavam atividades que possuem como eixo 0 planejamento, o desenvolvimento e a implementação de metodologias e ferramentas didáticas. Cicillini \& Silva, (2009), por exemplo, relatam a confecção, pelos professores participantes, de gibis e animações, que tinham como objetivo servir de modelos explicativos para fenômenos bio-físico-químicos. Segundo os autores, os temas trabalhados foram escolhidos por meio de discussões entre professores e pesquisadores.

É importante ressaltar que, durante a oferta de cursos mediados pelas TICs, nem todas as atividades se desenvolvem a distancia. Reis et al (2007) descrevem um curso que, embora utilizasse AVAs, teve como uma de suas estratégias pedagógicas a construção de experimentos que simulassem as constelações pelos alunos/professores. Os conteúdos nos quais as atividades eram baseadas e os roteiros para sua realização estavam disponíveis online em um sistema (REIS, SOUZA e BISCH, 2007). Segundo os autores, a atividade prática pode possibilitar a realização de um trabalho interdisciplinar, envolvendo, alem dos conteúdos científicos, as áreas de história, comunicação e artes, e habilidades necessárias durante o processo de planejamento.

Como apontado nesta revisão, de uma maneira geral, as iniciativas de formação continuada de professores têm adotado abordagens que reforçam o compartilhamento de saberes e experiências e oportunidades de reflexão-ação com a construção de atividades práticas e pautadas no cotidiano docente. Neste sentido, as TICs parecem constituir-se importantes aliadas pelo seu potencial de ampliar as formas de acesso e de representação do conhecimento e pelas diversas ferramentas de comunicação e colaboração.

\section{METODOLOGIA}

Neste estudo, foram analisados os ambientes virtuais de aprendizagem de seis cursos voltados para a formação continuada de professores de ciências da educação básica.

Estes cursos foram desenvolvidos por professores da área de ciências e ensino de ciências com o apoio da Constructore, uma ferramenta de autoria de cursos na internet desenvolvida pelo Laboratório de Tecnologias Cognitivas (NUTES/UFRJ) que tem como objetivo apoiar os professores no desenvolvimento, publicação e acompanhamento de recursos e atividades educativas semipresenciais ou à distância, sem a necessidade de conhecimentos de programação ou de suporte técnico especializado. Em especial, há um projeto que visa fortalecer a disseminação de cursos de extensão universitária no contexto das ciências da saúde e a Constructore tem sido utilizada neste sentido. Os cursos analisados foram desenvolvidos nos dois primeiros anos do projeto.

A análise dos cursos foi orientada pelo modelo proposto por Giannella \& Internet voltados para a área de ensino das ciências e da saúde. O modelo foi 
construído a partir de uma revisão da literatura acerca das diferentes classificações das formas de uso do computador e da Internet no ensino e contempla a análise das abordagens pedagógicas, das estratégias de ensino-aprendizagem e das modalidades pedagógicas de uso das TICs.

No que diz respeito às abordagens pedagógicas, o modelo propõe observar qual a tendência apresentada pelos materiais educativos, segundo o referencial de Kember e Kwan (2000): abordagem centrada na aprendizagem/no aluno, ou centrada no conteúdo/ professor. Como as autoras discutem, esta classificação não pressupõe a polarização das abordagens, mas a identificação de tendências, cujo enfoque pode se aproximar mais de uma proposta de ensino centrada na transmissão de informações ou da idéia de construção conjunta de conhecimento, numa parceria entre professores e alunos.

Para analisar as estratégias de ensino aprendizagem, o modelo contempla as categorias apresentadas por Schank e Cleary (1995) que tem como enfoque a área de ensino de ciências: aprender explorando, que ressalta estratégias que incentivem os alunos a buscarem conhecimentos em diferentes fontes, aprender fazendo, que enfatiza o uso de atividades práticas e reais; aprender refletindo, que ressalta a relevância de se criar oportunidades para os estudantes realizarem perguntas e questionamentos; aprendizagem baseada em casos, que explora a oferta integrada de diferentes perspectivas e olhares sobre um determinado caso/problema e aprendizagem incidental, que explora a utilização de atividades que não necessariamente tenham um enfoque educacional explícito.

Já a análise das modalidades de uso das TICs está baseada no conceito de Ciclo de Aprendizagem de Mayes e Fowler (1999). Segundo Giannella \& Struchiner (2010), estes autores definem três modalidades, a partir dos diferentes estágios de aprendizagem: Tecnologias primárias no estágio de conceituação, onde o enfoque está na oferta de material informativo; Tecnologias secundárias no estágio de construção, quando são propostas atividades de aprendizagem e Tecnologias terciárias no estágio de diálogo, quando são oferecidas possibilidades de interação entre os participantes.

As categorias e indicadores utilizados para analisar os cursos encontram-se sintetizados no quadro 1 , juntamente com os elementos presentes nos AVAs que foram analisados em cada curso. 
Quadro 1 - Categorias e indicadores utilizados para analisar os cursos de formação de professores oferecidos através da ferramenta Constructore, e elementos analisados em cada curso.

Categorias de análise

Abordagem

pedagógica

Estratégias de

ensino-

aprendizagem

Modalidades de uso das TICs
Indicadores

Elementos analisados nos cursos

Centrada no professor-conteúdo ou centrada no aluno-na aprendizagem (KEMBER e KWAN, 2000).

Oferta de recursos de informação e de atividades de fixação do conteúdo

Oferta de atividades que envolvem participação ativa do aluno: aprender explorando, aprender fazendo, aprender refletindo, aprendizagem baseada em casos e aprendizagem incidental

(SCHANK e CLEARY, 1995)

Estágios do ciclo de aprendizagem com o uso das TICs: estágio de conceituação (tecnologias primárias), estágio de construção (tecnologias secundárias) e estágio de diálogo (tecnologias terciárias) (MAYES e FOWLER, 1999).
Textos de apresentação dos cursos

(que contemplavam os objetivos, público-alvo, ementa etc), e de cada um dos módulos.

Enunciados das atividades e dos formulários de realização das atividades.

Textos das apresentações dos módulos

Enunciados das atividades propostas e dos formulários de realização das atividades.

Textos das apresentações dos módulos

Enunciados das atividades propostas e dos formulários de realização das atividades.

Recursos de comunicação 


\section{RESULTADOS}

O quadro 2 apresenta os seis cursos analisados. É interessante notar que todos os cursos tinham como enfoque aspectos relacionados às abordagens e estratégias de ensino/aprendizagem que são utilizadas pelos professores/alunos em sala de aula.

Como pode ser observado, a maioria dos cursos contava com a participação de mais de um professor, o que pode sugerir uma preocupação em oferecer apoio adequado aos alunos, já que os cursos, de uma maneira geral, apresentavam atividades que demandavam dialogo entre os participantes, além da realização de atividades práticas e reflexivas.

Entre os seis cursos apresentados, apenas C3 era totalmente à distância, enquanto os demais foram oferecidos na modalidade semipresencial de ensino. 
Quadro 2-Cursos de formação de professores de ciências oferecidos por meio da ferramenta Constructore.

Curso Objetivos
№ de №de professores alunos

$\begin{array}{ll}\begin{array}{l}\text { C1 Aplicação do método } \\ \text { científico em sala de aula }\end{array} & \begin{array}{l}\text { Instrumentalizar professores para a realização de } \\ \text { atividades em sala de aula baseadas na } \\ \text { metodologia científica }\end{array} \\ \text { C2 Brincando com a } & \begin{array}{l}\text { Oferecer noções sobre educação não formal em } \\ \text { ciência }\end{array} \\ \text { C3 Ciências.com } & \begin{array}{l}\text { Oferecer suporte aos professores do ensino } \\ \text { fundamental para complementar suas aulas com } \\ \text { atividades práticas que promovam a integração } \\ \text { com a teoria }\end{array}\end{array}$

7

professores do ensino fundamental e médio e pesquisadores da área de ensino de ciências por meio da socialização de experiências de práticas inovadoras em salas de aula de ciências, química, física e biologia.

C5 Instrumentalização Instrumentalizar professores e monitores para para realização de curso realização do curso "Aplicação do Método de férias Científico em Sala de Aula".

C6 Cotidiano escolar e Promover formação continuada de docentes e educação de jovens e profissionais de educação na temática EJA; adultos: múltiplos olhares

Desenvolver um espaço de troca de experiências entre a universidade e a educação básica acerca do ensino voltado para a promoção da saúde para jovens e adultos 
Com base no modelo de análise desenvolvido por Giannella \& Struchiner (2010), observou-se que cinco cursos contemplaram os três estágios do ciclo de aprendizagem de Mayes e Fowler (1999), e um (C3) concentrou-se no estágio de conceituação.

O curso C3 concentrou-se no estágio de conceituação, já que a estratégia de ensino-aprendizagem adotada baseava-se na oferta de recursos de informação. Este curso, que apresentou abordagem pedagógica centrada principalmente no conteúdo (KEMBER e KWAN, 2000), tinha uma dinâmica auto-instrucional, disponibilizando roteiros de atividades experimentais para que os alunos (professores de ciências da educação básica) pudessem aplicar com seus estudantes em sala de aula. Assim, o foco de C3 foi o estágio de conceituação proposto por Mayes \& Fowler (1999), já que os roteiros foram disponibilizados, mas não houve uma proposta de reflexão acerca dos experimentos ou de formas de utilizá-los em sala de aula.

\section{O objetivo do curso é oferecer suporte on line aos professores de ciências de ensino fundamental que desejam complementar suas aulas com práticas interessantes". Aqui, você poderá encontrar instruções passo-a-passo, com links para fotos e vídeos, de experimentos de ciências. (C3 Apresentação do curso)}

Os cursos C1 e C5, apesar de não estarem concentrados apenas no estágio de conceituação, disponibilizaram diversos roteiros de experimentos científicos, acompanhados dos principais conceitos científicos envolvidos em seus resultados. Já nos cursos C2, C4 e C6, foram encontrados materiais, principalmente textos e slides, contendo propostas de estratégias inovadoras voltadas para o ensino de ciências, além de reflexões acerca da metodologia didática tradicional comumente vigente nas escolas.

Em relação ao segundo estágio de aprendizagem (construção), foram identificadas quatro diferentes estratégias de ensino-aprendizagem, segundo as categorias de Schank e Cleary (1995): aprender explorando, aprender fazendo, aprender refletindo e aprendizagem incidental.

A estratégia "aprender explorando" foi identificada em dois cursos (C5 e C6), que tinham propostas de atividades que estimulavam os estudantes a ampliar as fontes de aprendizagem além dos recursos oferecidos no curso. No curso C5, por exemplo, os alunos deveriam buscar conceitos que servissem como base para propostas de experimentos.

No segundo módulo, o cursista deverá buscar bibliografia, em fontes confiáveis, para que os fundamentos teóricos dos experimentos sejam abordados de forma adequada. (C5 Apresentação do curso).

Quatro cursos apresentavam atividades que ofereciam a oportunidade de os alunos aprenderem na ação, ou na experimentação de um determinado processo (aprender fazendo) (C1, C2, C5 e C6). Nestes cursos, observou-se a intenção de ancorar os saberes práticos aos saberes teóricos. No curso C2 a estratégia "aprender fazendo" foi identificada na realização de atividades práticas presenciais, como oficinas para a construção de aparatos didáticos utilizados para explicar conceitos científicos. 
Após uma breve apresentação contextualizada sobre a temática do mês é realizada uma oficina na qual o público terá a oportunidade de discutir, aprender e construir um aparato referente a um tema relacionado à Ciência. (C2 Apresentação do módulo Faça você mesmo).

Já o curso C6 propunha a construção de um portfólio virtual, pelo qual os professores/alunos relatavam experiências vivenciadas durante as aulas presenciais, além de reflexões e percepções acerca das leituras realizadas e das discussões no fórum. Esse portfólio serviu como uma base para a auto-avaliação, na medida em que possibilitou o acompanhamento do processo de aprendizagem e incluiu aspectos relacionados a uma possível contribuição/influencia do curso sobre as atividades profissionais do professor.

\section{As atividades do curso, aulas presenciais, leituras, formulários e fóruns proporcionaram experiências que serão relatadas em um portfólio virtual. Leia o instrumento de avaliação e poste suas dúvidas (C6 Atividade do modulo Construindo um Portfólio)}

Oferecidos pelo mesmo grupo de professores da Universidade, os cursos $\mathrm{C} 1 \mathrm{e}$ C5 apresentavam uma abordagem pedagógica semelhante, pois ambos possuíam como eixo norteador uma atividade que os alunos deveriam realizar ao longo de cada curso. Por exemplo, C1 consiste em um curso de férias construído com o objetivo de promover estratégias educativas baseadas na metodologia cientifica pelos professores. A proposta central, neste caso, é o desenvolvimento de uma atividade respaldada no método científico para ser trabalhada em sala de aula.

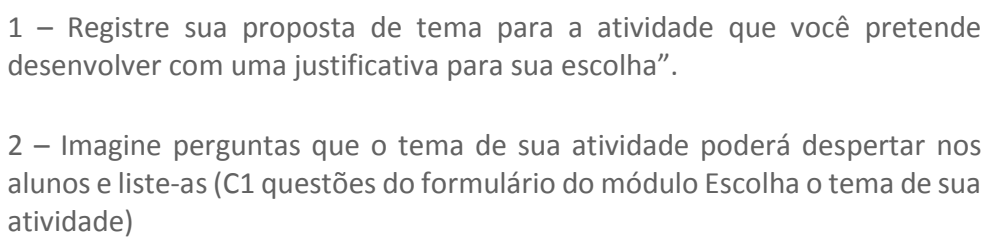
alunos e liste-as (C1 questões do formulário do módulo Escolha o tema de sua atividade)

E com o objetivo de qualificar professores para a multiplicação deste curso de férias, C5 possui como foco o planejamento, por professores que já cursaram C1, de um curso de férias para ser aplicado com outros professores de ciências da educação básica.

O cursista deverá usar a criatividade para escolher um tema para montar o curso de férias(C5 Apresentação do curso)

Nestes cursos, C1 e C5, todos os materiais e recursos disponibilizados e as discussões realizadas foram utilizados para mediar e apoiar a conclusão dessa atividade principal.

A estratégia de aprendizagem "aprender refletindo", que estimulava a reflexão dos alunos sobre textos, atividades e discussões, foi encontrada em quatro cursos (C1, C2, C4, e C5). O curso C4, por exemplo, tinha como foco principal o raciocínio e a troca de experiências acerca de questões relacionadas à implementação de inovações educativas: 
Nessa atividade você terá que selecionar uma das inovações postadas e listar as dificuldades e as soluções encontradas para a implementação da inovação em seu contexto escolar. Poste sua contribuição no fórum, pois assim poderemos trocar informações e experiências com todos. (C4 Atividade do módulo Obstáculos e soluções para implementação da inovação).

Nos cursos $\mathrm{C} 1$ e $\mathrm{C} 5$, os alunos deveriam escolher um tema para o planejamento de: a) uma atividade baseada no método científico (no caso do curso C1), e b) um curso de férias a ser planejado e desenvolvido (no caso do curso C5). Assim, propunha-se uma reflexão acerca dos temas e das possibilidades a serem desenvolvidas a partir dessas escolhas, verificando sua adequação aos objetivos do curso.

(...) o cursista deverá escolher um tema contido no currículo programático do(s) anos(s) escolar(es) em que leciona, para montar a atividade prática (...). Em seguida, a partir do tema escolhido, elaborar uma lista de perguntas possíveis de serem feitas por seus alunos (C1 Apresentação do curso)

Neste módulo, espera-se que você escolha um tema para a realização de um Curso de férias levando em conta os interesses, as competências e habilidades do público-alvo, além da infra-estrutura e recursos disponíveis no local da realização do curso (C5 Apresentação do módulo Escolha do tema do seu curso de férias).

Já no curso C2, foi proposto aos participantes a redação de um relatório, reunindo suas impressões acerca da visita a um museu

\begin{abstract}
Esta atividade implica na confecção de um relatório (...) onde o aluno deve incluir suas impressões acerca da visita. Essas impressões devem versar sobre o conteúdo, forma de veiculação deste conteúdo, pontos que o agradaram, pontos que não o agradaram e possibilidades de contextualização com sua realidade (C2 Apresentação do módulo Visita a um museu).
\end{abstract}

A estratégia denominada "aprendizagem incidental", foi encontrada no curso C6, que tinha como objetivo o compartilhamento de experiências entre professores e pesquisadores universitários. Este curso utilizava filmes para promover a reflexão e a discussão acerca de questões relacionadas à promoção de saúde de alunos da modalidade EJA.

Visitando o portal do Envelhecimento, nos links disponibilizados, o participante deve escolher um filme, assisti-lo e apresentar como a representação social da velhice é abordada no curta/longa metragem (C6 Atividade do módulo Velhice, Saúde, Educação e Representações Sociais.)

O terceiro estágio do ciclo de ensino-aprendizagem, "diálogo" foi identificado em quatro cursos (C1, C4, C5 e C6). Nestes casos, a utilização dos recursos de comunicação incluía o compartilhamento das experiências e dos resultados das tarefas realizadas no fórum de discussão, promovendo a colaboração entre os participantes envolvidos por meio da ferramenta constructore. No curso C4, por exemplo, o fórum foi amplamente utilizado, na medida em que os estudantes deviam propor estratégias inovadoras em sala de aula, compartilhar e discutir com seus colegas e professores. 
Proporcionar um espaço de discussão e troca entre professores do ensino fundamental e médio e pesquisadores da área de ensino de ciências por meio da socialização de experiências de práticas inovadoras em salas de aula de Ciências, Biologia, Física e Química (C4 Apresentação do curso).

Já no curso $\mathrm{C} 1$, os participantes utilizavam o fórum de discussão para expôr experimentos previamente planejados, que eram debatidos entre os colegas e remodelados. A partir disso, discutiam-se formas de aplicação do material em sala de aula, incluindo possibilidades de abordagem e discussões de resultados.

\footnotetext{
Durante toda a atividade, as idéias e propostas do cursista, postadas em fórum, serão discutidas pelos colegas e professores e tutores do curso, com o objetivo de orientá-lo na condução das tarefas (C1 Apresentação do curso)
}

\section{DISCUSSÃO}

A maioria dos cursos analisados neste estudo promovia uma abordagem de ensino e aprendizagem baseada no aluno/aprendizagem $(=5)$, com atividades voltadas para a construção e o planejamento de materiais ou metodologias de ensino inovadoras. Este resultado é compatível com os cursos encontrados na revisão bibliográfica, o que pode significar uma tentativa de favorecer o necessário empoderamento dos professores para que estes possam refletir sobre suas práticas e modificá-las, a fim de melhorar o processo de aprendizagem dos alunos, como apontado por Tessio et al (2005). Concordamos com Lima \& Pinheiro (2005), que a utilização de abordagens ativas e dialógicas em cursos de formação de professores é fundamental para que estes possam incorporar estratégias semelhantes em suas práticas.

Com relação ás estratégias de ensino e aprendizagem, a oferta de recursos de conteúdo estava presente em todos os cursos analisados, e está relacionada, segundo Mayes \& Fowler (1999), ao estágio de conceituação, sendo encontradas sob duas perspectivas diferentes: a) disponibilização de materiais científicos, e b) disponibilização de materiais contendo reflexões acerca de práticas pedagógicas. Como apontado por diversos autores, a aquisição de conceitos científicos serve como base para que os professores possam realizar diferentes atividades educativas com seus alunos (AIRES \& LAMBACH, 2009, NAVAS, MALACHIAS, NUNES e FEJES, 2005; NOVELLO, LAURINO e MALACHIAS, 2005). Com relação aos conhecimentos pedagógicos, Silva e Schnetzler (2001) afirmam que os professores precisam desenvolver a capacidade de questionar estratégias tradicionais de ensino, concebendo a prática pedagógica como objeto de investigação, além de planejar atividades didáticas. Para isso, materiais que levem os docentes à reflexão e a discussão podem apoiar iniciativas de formação continuada de professores. Para essa estratégia de ensino, as TICs apresentam grande contribuição, devido à facilidade de organização e disponibilização de materiais, que possibilita o acesso dos alunos à meios de informação variados, de acordo com a necessidade $\mathrm{e}$ interesse (NAVAS, MALACHIAS, NUNES e FEJES, 2005).

Segundo Giannella (2007) e Giannella e Struchiner (2010), a oferta de recursos de conteúdo relaciona-se com a categoria "acesso e organização da informação" proposta por Sugrue (2000). Segundo a autora, essa modalidade ressalta a 
construção de modelos não lineares de conhecimento, compatíveis com o sistema de memória e raciocínio humano (SUGRUE, 2000).

Além da disponibilização de recursos de conteúdo, as atividades que envolvem participação ativa do aluno foram identificadas em cinco cursos. Esse quadro pode estar relacionado às atuais exigências da formação de professores, que torna necessário ao professor refletir e avaliar continuamente sua prática, a fim de utilizar metodologias de ensino que auxiliem os estudantes na construção de conhecimento (BONZANINI e BASTOS, 2009; CICILLINI e SILVA, 2009; MOITA, CANUTO e SILVA, 2011), e se capacitar para realizar diferentes atividades em sua estratégia de ensino (ARROIO, DINIZ e GIORDAN, 2005). Diversos autores apontam que essas práticas são importantes para superar o ensino baseado na simples transmissão de informações (GABINI e DINIZ, 2005; GEREMIAS, CIMA e ANGOTTI, 2005; MOITA, CANUTO e SILVA, 2011). A estratégia "aprender explorando" foi identificada em três cursos, ressaltando-se que os recursos tecnológicos podem possibilitar a exploração e a busca de informações pelos professores/alunos. Geremias et al (2005) relatam um curso no qual os alunos eram estimulados a buscar informações na internet a partir do que foi disponibilizado previamente. Segundo eles, com o auxílio dos professores e de colegas, os estudantes tem a oportunidade de aprender a procurar e selecionar informações confiáveis e relevantes para seu aprendizado.

Além disso, em quatro cursos observamos a intenção de ancorar os saberes práticos aos saberes teóricos, por meio da estratégia "aprender fazendo". Segundo Reis et al (2007), a confecção de materiais específicos pelos professores usuários envolve a exploração dos diversos conceitos e conhecimentos envolvidos na sua elaboração. Isso estimula a aplicabilidade do material em sala de aula, na medida em que o professor conhece melhor o assunto em questão (REIS, SOUZA e BISCH, 2007). Essa estratégias é reiterada por Gabini e Diniz (2007), que apontam que o desenvolvimento de atividades que promovam a melhoria nos processos de ensino/aprendizagem aproxima os docentes desse processo, tendo em vista a oportunidade de relacionar propostas didáticas inovadoras com seus contextos de trabalho.

A estratégia "aprender refletindo" foi utilizada em cinco cursos, como no curso C4, por exemplo, que tinha como objetivo "proporcionar um espaço de discussão e troca entre professores do ensino fundamental e médio e pesquisadores da área de ensino de ciências por meio da socialização de experiências acerca de práticas inovadoras em salas de aula de Ciências". De forma semelhante, Cicillini \& Silva (2009) descrevem a formação de Núcleos de trabalho entre professores, tutores e pesquisadores, afirmando que o incentivo ao diálogo e à reflexão por parte dos professores estimula-os a perceber suas capacidades para a construção de projetos de ensino inovadores. Segundo Schon (2000), a formação docente deve capacitar o professor a refletir criticamente sobre suas ações, avaliando os problemas que surgem no decorrer do seu trabalho em sala de aula e tomando decisões.

Além disso, um curso utilizou a estratégia "aprendizagem incidental", oferecendo recursos tradicionalmente não educativos para motivar e promover a discussão de questões relacionadas ao tema trabalhado no curso. Segundo Schank \& Cleary (1995), essa estratégia estimula a construção de conhecimento, na medida em que transforma determinados fatos ou discussões em matéria prima para o processo de aprendizagem. 
O "estágio de diálogo", relacionado aos "estágios do ciclo de aprendizagem, estava presente em quatro cursos, oportunizando o diálogo, a interação e a troca de experiências e reflexões entre os participantes. Neste caso, é observado o papel do uso das TICs para a aprendizagem colaborativa, estimulando os professores a se formarem como profissionais coletivos, por meio do diálogo e da reconstrução da identidade profissional (LIMA e PINHEIRO, 2005). Diversos autores defendem que esta modalidade enfoca a participação ativa e interativa de todos os atores envolvidos no processo educacional (GIANNELLA, 2007; ROGOFF, 1998). Diante da diversidade de ferramentas comunicacionais oferecidas pela internet, um dos grandes potenciais dos AVAs é a construção de comunidades colaborativas, que explorem as diferentes especialidades e conhecimentos de seus membros, enriquecendo e diversificando a atividade educativa (GIANNELLA, 2007; SUGRUE, 2000). Segundo Gabini e Diniz (2007) as atividades de formação docente devem ser realizadas por meio de um trabalho coperativo, que leve em conta o contexto, o conhecimento prévio dos professores em exercício e as atividades que pretendem desenvolver.

\section{CONCLUSÕES:}

A partir da análise realizada, pôde-se caracterizar as abordagens pedagógicas mais utilizadas em cursos de formação continuada de professores de ciências da educação básica, como centradas mais no aluno/na aprendizagem. Essa tendência é constatada por meio das atividades propostas, que tinham como foco central o planejamento, o desenvolvimento e a aplicação de metodologias de ensino, a partir da realidade dos participantes.

Além disso, destaca-se a utilização de estratégias de construção do conhecimento pelo professor-aluno, como "aprender explorando", "aprender fazendo", "aprender refletindo" e "aprendizagem incidental" auxiliada pela disponibilização de materiais pedagógicos que integram teoria e prática, e pelas formas de comunicação entre os sujeitos, que apontam para um trabalho colaborativo.

Compreendemos que os materiais educativos analisados são produtos da ação dos professores/pesquisadores, constituindo se como lentes para analisar suas opções pedagógicas (STRUCHINER e GIANNELLA, 2006), na medida em que refletem suas visões acerca do trabalho e do papel do professor de ciências na educação básica.

A aplicação deste modelo de análise aponta também uma ampla utilização dos recursos que podem ser oferecidos em um AVA para apoiar iniciativas de formação continuada de professores da educação básica. A possibilidade de disponibilizar ferramentas de comunicação e atividades que estimulam os professores/alunos a refletir sobre suas práticas e implementar novas estratégias de ensino estiveram articuladas com os objetivos dos cursos analisados, em estimular práticas pedagógicas inovadoras, que rompam com o atual modelo de ensino baseado na transmissão de informações.

Além disso, a abertura de novos canais de comunicação auxilia no estabelecimento da aprendizagem colaborativa entre professores e pesquisadores, estimulando a superação de práticas de ensino tradicionais. Ressalta-se que a utilização de ferramentas tecnológicas pode auxiliar os 
professores a alterar a realidade centrada nas aulas expositivas na medida em que esses recursos são integrados de forma natural ao cotidiano (GABINI e DINIZ, 2007). Nesse contexto, a parceria entre universidade e escola apresenta um importante papel social, na medida em que os diferentes saberes podem se complementar durante o desenvolvimento e a implementação de estratégias e atividades educativas inovadoras. 


\title{
Pedagogical approaches, teaching and learning strategies, and modalities of information and communication technologies use: analysis of courses offered to elementary science teachers
}

\begin{abstract}
This study aymed at analysing Virtual Learning Environments offered to elementary science teachers. Using an analytical framework for internet-based science educational material, we examined pedagogical approachs, teaching and learning strategies and modalities of ICTs' use in six courses. Five courses presented student/learning centered pedagogical approach, integrating strategies such as: learning by exploring (C2, C5 e C6), learning by doing ( $C 1, C 2, C 5$ e C6), learning by reflection (C1, C4, C5 e C6) and incidental learning (C6). One course presented teacher/teaching centered pedagogical approach. The courses explored ICT potential for: information acess and organization, authentical activities and collaborative learning. Based on the annalisys, we can observe that ICT use in teacher continuing education has facilited adoption of active and collaborative learning approaches, contibuting to improve teaching.
\end{abstract}

KEYWORDS: Virtual learning environment annalisys. Teacher Continuing Education. Information and Communication Technologies. 


\section{REFERÊNCIAS}

AIRES, J. A.; LAMBACH, M. Contextualização do ensino de química pela problematização e alfabetização científica e tecnológica: uma experiência na formação continuada de professores. Anais do VII Encontro Nacional de Pesquisa em Educação em Ciências, Florianópolis, SC, Brasil, Nov/2009.

ARROIO, A.; DINIZ, M. L e GIORDAN, M. A utilização do vídeo educativo como possibilidade de domínio da linguagem áudio-visual pelo professor de ciências. Anais do V Encontro Nacional de Pesquisa em Educação em Ciências, Bauru, SP, Brasil, Nov/2005

BARRETO, R. G. Tecnologia e Educação: trabalho e formação docente. Educação \& Sociedade, v. 25, n. 89, p. 1181-1201, set-dez/2004

BONZANINI, T. K. e BASTOS, Formação continuada de professores de ciências: algumas reflexões. Anais do VI Encontro Nacional de Pesquisa em Educação em Ciências, Florianópolis, SC, Brasil, dez/2007.

CICILLINI, G. A. e SILVA, E. P. Q. A formação de professores em acontecimentos: a produção dos saberes escolares nas ciências naturais no nível médio em Uberlândia - MG. Anais do VII Encontro Nacional de Pesquisa em Educação em Ciências, Florianópolis, SC, Brasil, Nov/2009.

GABINI, W. S. e DINIZ, R. E. S. A informática como estratégia didática no ensino de química. Anais do V Encontro Nacional de Pesquisa em Educação em Ciências, Bauru, SP, Brasil, dez/2005.

GABINI, W. S. e DINIZ, R. E. S. Formação continuada de professores: integrando a análise de softwares educativos de química a esse processo. Anais do VI Encontro Nacional de Pesquisa em Educação em Ciências, Florianópolis, SC, Brasil, dez/2007.

GIANNELLA, T. R. Inovações no ensino das ciências e da saúde: pesquisa e desenvolvimento da ferramenta Constructore e do Banco virtual de neurociência. Rio de Janeiro, 2007. Tese (Doutorado em química biológica Educação, Difusão e Gestão em Biociências) - Instituto de Bioquímica Médica, Universidade Federal do Rio de Janeiro, 2007. 
modelo de análise de materiais educativos baseados na internet. Revista Electronica de ensenanza de las ciências, v. 9, n. 3, p. 530-548, 2010.

GEREMIAS, B. M.; CIMA, V. A. e ANGOTTI, J. P. A. Contribuições dos meios tecnológicos: comunicativos para o ensino de ciências. Anais do V Encontro Nacional de Pesquisa em Educação em Ciências, Bauru, SP, Brasil, dez/2005

KEMBER, D. e KWAN, K. Lecturers Approaches to teaching and their relationships to conception of good teaching. Instructional Science, v. 28, n. 5, p. 469-490, 2000 .

LIMA, A. C. C. e PINHEIRO, S. C. Tecnologias da informação e comunicação na formação de professores de ciências. Anais do V Encontro Nacional de Pesquisa em Educação em Ciências, Bauru, SP, Brasil, dez/ 2005.

MAYES, J. T. e FOWLER, C. J. H. Learning Technology and Usability: a framework for understanding courseware. Interacting with computers, v. 11, n. 5, p. 485497, 1999

MENDEZ M. I. P. e FIGUEIROA, S. F. M. A terra sob medida: debates da história da ciência em espaços virtuais de aprendizagem. Anais do V Encontro Nacional de Pesquisa em Educação em Ciências, Bauru, SP, Brasil, dez/2005.

MISHRA, P. e KOEHLER, M. J. Not "what" but "how": Becoming design-wise about educational technology. In_ZHAO, Y. (Ed.). What should teachers know about technology? Perspectives and practices. Greenwich: information age publishing, p. 99-121, 2003.

MOITA, F. M. G. S. C.; CANUTO, E. C. A. e SILVA, A. Formação continuada de professores de ciências: uma reflexão sobre os possíveis impactos dos indicadores do IDEB. Anais do VIII Encontro Nacional de Pesquisa em Educação em Ciências, Campinas, SP, Brasil, dez/2011.

NAVAS, A. M.; MALACHIAS, M. E. I.; NUNES, C. A. A. e FEJES, M. E. Representações de professores sobre simulações e animações: uma primeira aproximação através do projeto LabVirt Química. Anais do V Encontro Nacional de Pesquisa em Educação em Ciências, Bauru, SP, Brasil, dez/2005.

NOVELLO, T. P.; LAURINO, D. P. e RODRIGUES, S. C. Dinâmica de interação no ambiente virtual Mathemolhes. Anais do V Encontro Nacional de Pesquisa em Educação em Ciências, Bauru, SP, Brasil, dez/2005. 
REIS, J. C.; SOUZA, S. R. e BISCH, S. M. Reconhecimento do céu na formação continuada de professores do ensino fundamental em Ciências. Anais do VI Encontro Nacional de Pesquisa em Educação em Ciências, Florianópolis, SC, Brasil, nov/2007.

ROGOFF, B. Observando a atividade sociocultural em três planos: Apropriação participatória, participação guiada e aprendizado. In_WERTSCH, J.; RIO, P; e ALVARES, A. Estudos socioculturais da mente. Porto Alegre: Artmed, 1998, p. 123-142.

SCHANKY, R. e CLEARY, C. Engines for education. Hillsdale, NJ: Lawrence Erlbaun Associates, 1995

SCHON, D. A. Educando o profissional reflexivo, um novo design para o ensino e a aprendizagem. Trad. Roberto Cataldo Costa. Porto Alegre: Artmed, 2000.

SUGRUE, B. Cognitive approaches to web-based instruction. In: S. Lajoie (Ed), Computer has cognitive tools: no more walls, (pp. 162-163). Mahwah, New Jersey, Lawrence Erlbaun Associates, 2000.

STRUCHINER, M. e GIANNELLA, T. R. Novas tecnologias de Informação e Comunicação na formação de recursos humanos em saúde. In: S. Monteiro \& E. Vargas (org.), Educação, Comunicação e tecnologia educacional: interfaces com o campo da saúde, (pp. 129-140), Rio de Janeiro, RJ: FioCruz, 2006

TESSIO, N. M.; REMBADO, F. M.; SANTOS, B. F. e PORRO, S. Empleo de um espacio virtual para El trabajo colaborativo en La articulación universidad-escuela hacia um mejoramento em la formacion de professores en el área de ciências naturales. Anais do V Encontro Nacional de Pesquisa em Educação em Ciências. Anais do VI Encontro Nacional de Pesquisa em Educação em Ciências, Bauru, SP, Brasil, dez/2005.

ZAPPAROLI, F. V. D.; BUENO, E. A. S. e ARRUDA, S. M. A utilização da experimentoteca na formação continuada de professores. Anais do V Encontro Nacional de Pesquisa em Educação em Ciências, Bauru, SP, Brasil, dez/2005. 
Aprovado: 06 mai. 2016

DOI: $10.3895 /$ rbect.v9n1.2952

\section{Como citar:}

WARDENSKI, R. F.; STRUCHINER, M.; GIANNELLA, T. R. Abordagens pedagógicas, Estratégias de ensinoaprendizagem e Modalidades de uso de Tecnologias de Informação e Comunicação: uma Análise de Cursos para Formação Continuada de Professores de Ciências. Revista Brasileira de Ensino de Ciência e

Tecnologia, v. 9, n. 1, p. 407-427, jan./abr. 2016. Disponível em:

<https://periodicos.utfpr.edu.br/rbect/article/view/2952>. Acesso em: xxx.

Correspondência:

Rosilaine de Fátima Wardenski

Av Carlos Chagas Filho, 373, Centro de Ciências da saúde, Bloco L, sala 32, Rio de Janeiro, Rio de Janeiro. Miriam Struchiner

Av Carlos Chagas Filho, 373, Centro de Ciências da saúde, Bloco L, sala 32, Rio de Janeiro, Rio de Janeiro. Tais Rabetti Giannella

Av Carlos Chagas Filho, 373, Centro de Ciências da saúde, Bloco L, sala 32, Rio de Janeiro, Rio de Janeiro. Direito autoral: Este artigo está licenciado sob os termos da Licença Creative Commons-Atribuição 4.0 Internacional.

\section{(c) (1)}

